\title{
Adaptive Self-Embedding Scheme with Controlled Reconstruction Performance
}

\author{
Paweł Korus, Member, IEEE, Andrzej Dziech
}

\begin{abstract}
In this study we addresses the problem of adaptive self-embedding, where the reconstruction quality is controlled individually for different fragments of a digital image. We focus on the impact of incorporating content adaptivity features on the restoration success conditions, and the achievable reconstruction performance. We analyze the problem theoretically, and validate the obtained results experimentally with a fully functional selfembedding scheme. Our analysis shows that introduction of multiple reconstruction profiles, even with significantly lower restoration fidelity, does not need to improve the achievable tampering rate bounds. The obtained fine-grained control over the reconstruction process is exploited to provide guarantees on certain performance aspects. Based on the derived theoretical model, we propose a procedure for optimization of the overall reconstruction quality given constraints on the desired target tampering rate, and the required quality level for selected image fragments. Such guarantees are of principal importance in a number of applications.
\end{abstract}

Index Terms-Content Authentication; Content Reconstruction; Self-Embedding; Digital watermarking; Content Adaptation

\section{INTRODUCTION}

Self-embedding allows for incorporating content reconstruction features into digital image authentication schemes [1]. In addition to content hashes for authentication purposes, a lowquality version of the original image is embedded into the image itself as a digital watermark. Upon identifying the tampered image fragments, their approximate original appearance is restored from the embedded reconstruction reference.

The performance of self-embedding schemes is commonly expressed by means of objectively measured reconstruction quality, and restoration conditions. The former is typically given as a peak signal to noise ratio (PSNR), and the latter as a bound on the allowed amount of modifications, i.e., the tampering rate. These two performance aspects are tightly connected, and the trade-off between them needs to be properly balanced.

Copyright (c) 2013 IEEE. Personal use of this material is permitted. However, permission to use this material for any other purposes must be obtained from the IEEE by sending a request to pubs-permissions@ieee.org.

P. Korus, A. Dziech are with the Department of Telecommunications of the AGH University of Science and Technology, al. Mickiewicza 30, 30 059 Kraków, Poland, fax: +48 12 6342372. Telephone: +48 126173805 (P. Korus), +48 126172616 (A. Dziech), E-mail: pkorus@agh.edu.pl, dziech@kt.agh.edu.pl

The research leading to these results has received funding from the INDECT project funded by European Community's Seventh Framework Programme (FP7 / 2007-2013) under grant agreement no.[218086] and partly from the INSIGMA project funded from the European Regional Development Fund, project no. POIG.01.01.02-00-062/09.
The most recent research on self-embedding focuses on the techniques for exploiting this quality-robustness tradeoff. There exist two fundamental approaches to the problem, i.e., flexible [2], [3], and adaptive restoration techniques [4], [5], [6], [7], [8]. In the former, the reconstruction quality deteriorates uniformly for the whole image as the tampering rate grows. High-quality reconstruction is possible, provided that the tampering affects only a small image area.

In adaptive schemes the reconstruction quality is controlled for each image fragment individually, and does not change with the tampering rate. Typically, such schemes define several reconstruction profiles, which determine how a single image block is represented, and reconstructed. The profiles are usually dedicated to different types of content, and feature various restoration fidelity. As a result, individual image fragments can be represented in a way which best matches their content.

Initially, adaptive self-embedding was seen just as a technique for reducing the length of the reference bit-stream by allowing flat image blocks to be represented by shorter streams. Some of the defined profiles can have a marker role, which designates their blocks for reconstruction with a different method, e.g., using inpainting [9], or completely excludes them from the reconstruction process [4]. The resulting shorter bit-stream is essentially a means of improving the achievable tampering rates.

In our opinion, the primary benefit from employing adaptivity features is the additional degree of freedom, which allows for precise control over the inherent restoration trade-offs [4]. In particular, it becomes possible to provide guarantees for certain aspects of the restoration process. For instance, selected image fragments, representing the most important content, can always be restored with high quality, at the expense of less valuable background content. Such features are of principal importance in a number of applications, e.g., for protection of video surveillance footage, where details like faces, or license number plates should be regarded as high-priority content.

The primary goal of this study is to design a mechanism capable of providing guarantees for both the fidelity of selected image fragments, and the achievable tampering rates. For this purpose, we perform theoretical analysis of the adaptive content reconstruction problem. All theoretical results are verified experimentally with a fully functional self-embedding scheme. The starting point for our discussion is a recently proposed model of the self-recovery problem [10]. The model formulates the reconstruction as a communication over an erasure channel, and gives a closed-form expression for the achievable success bounds for traditional self-recovery with uniform image quality. In this work, we extend the model 
to take into account content adaptivity features. Variation of the number of reference bits on a per-block basis introduces new challenges, and requires to consider differences in the tampering order. The proposed approach allows for accurate modeling of the behavior of even complex adaptive schemes.

Based on the performed analysis, we propose an algorithm for computation of the optimal assignment of the reconstruction profiles to individual image blocks. The procedure aims to maximize the overall reconstruction quality, given constraints on the quality of selected fragments, and the target tampering rate. In addition to the performance guarantees, such an approach can deliver better reconstruction efficiency, compared to traditional uniform-quality restoration. However, despite significantly shorter reference streams, introduction of reconstruction profiles with lower fidelity does not necessarily improve the achievable tampering rates.

This paper is organized as follows. Section II gives a brief overview of the history, and the directions of the selfembedding research. The necessary notation, a formal statement of the problem, and the operation of the considered selfembedding scheme are presented in Section III. The theoretical analysis of the adaptive reconstruction problem is performed in Section IV. The designed procedure for reconstruction profile assignment is presented in Section V. The results of experimental evaluation are discussed in Section VI. We conclude in Section VIII.

\section{RELATED WORK}

The concept of self-embedding has been first introduced in [1]. In the proposed method, a compressed representation of each image block is embedded by means of least significant bit substitution (LSBS) into a different block. The utilized compression method resembles traditional JPEG, and produces a bit-stream of length equal to the embedding capacity. Tampered blocks are then recovered from their reference information, provided that their carrying block remained authentic.

In [11] the authors formulate content reconstruction as an irregular sampling problem. The restoration is performed by iterative projections onto convex sets. The reference information is obtained by logical exclusive disjunction on block-based cosine transform coefficients' polarity and pseudo-random bit sequences. The watermark is embedded by modulating middle frequency components of the pinned sine transform. Although the method provides very low reconstruction quality, it can survive mild image processing, including lossy compression, and Gaussian filtering.

Initially, the reconstruction quality was treated as a secondary aspect, and it used to be sufficient for the restored content to be barely discernible for a human observer. Recently, it has been noticed that certain applications require highquality reconstruction. The scheme from [12] features lossless reconstruction by using difference expansion, a reversible watermarking technique, for information embedding. While the watermarked images are severely distorted, having PSNR of approximately $29 \mathrm{~dB}$, the decoder can recover the original, unwatermarked images. The main disadvantage of the scheme is limited robustness against malicious modifications. The maximum supported tampering rate is only $3.2 \%$.
Successful reconstruction of extensive tampering was demonstrated in [13]. At the cost of low reconstruction quality, the scheme allows for tampering up to $59 \%$ of the image area. The reference information is obtained by coarse quantization of selected low-frequency DCT coefficients. In order to spread the reference information over the entire image, individual blocks are pseudo-randomly grouped into pairs, and the reference information is projected onto the watermark payload using a random binary matrix. The utilized embedding technique is LSBS with embedding payload of 3 bits per pixel.

In order to provide support for both high-quality reconstruction and robustness against extensive tampering, flexible schemes have recently been introduced. When only small image fragments are tampered, they can be restored with high fidelity, which is systematically reduced as the tampering rate grows. The scheme from [14] defines a scalable reference stream, where each quality level can survive different tampering. The reference information is obtained from a wavelet-like decomposition of the image blocks.

In [3] the authors propose to use compressive sensing for content reconstruction. The reference information is obtained by randomly sampling small groups of scattered DCT coefficients. Depending on the number of missing coefficients within each group, they can be restored either with a proposed compositive reconstruction mechanism, or with compressive sensing. The reconstruction fidelity deteriorates gradually, as the restoration assumes approximate character for growing uncertainty within the groups.

In the flexible reconstruction scheme from [2], 5 most significant bits (MSB) of two randomly selected pixels are combined with exclusive disjunction, then grouped with other pixel pairs for embedding in randomly selected image blocks. Depending on the authenticity of individual pixels, the MSBs of a pixel pair can be either fully or partially recovered. The remaining uncertainty is resolved by exploiting local pixel correlations.

Virtually all of existing self-embedding schemes are characterized by uniform reconstruction quality. In flexible schemes the quality still drops uniformly for the whole tampered area. Even content adaptivity features were motivated by the possibility to shorten the representation of low-texture fragments, but without noticeable fidelity impact. While such an approach is not amiss by principle, it can be taken a step further. Certain applications could vastly benefit from performance guarantees both in terms of quality of selected regions, and the desired robustness against tampering. These requirements can be met by adjusting the reconstruction of less important background content [4]. This observation motivates the research presented in this study.

\section{AdAPtive SElF-EMbEDding Scheme}

Adaptive self-embedding differs from traditional schemes by using multiple reconstruction profiles. The profiles instruct the system's entities how to describe, and restore the content of individual image blocks. In this study, the reconstruction reference is generated from a block-based discrete cosine transform (DCT) spectrum. A profile is defined by a set of 


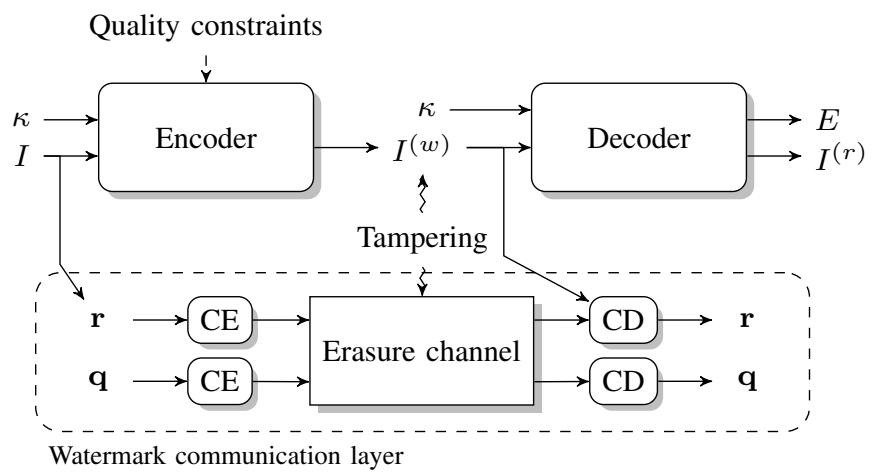

Fig. 1: General operation of adaptive self-embedding; communication of the watermark payload through an erasure channel: CE - channel encoder, CD - channel decoder.

quantization code-books, and the allocation of the precision of coefficient representation.

The mapping between image blocks and the reconstruction profiles is referred to as a quality descriptor $\mathbf{q}$, and it needs to be reliably communicated to the decoder. In this study, the descriptor is separated from the reference information. Alternatively, information about the assigned profile could be placed in a header of the reference information of each individual block [6].

In this study, we consider an adaptive self-embedding scheme, which extends the reference scheme described in [10]. In order to facilitate easier reading, we introduce the basic notation and operation principles, originally used in [10]. Fig. 1 illustrates the communication of the watermark information between the encoder and the decoder. The former produces a protected image $I^{(\mathrm{w})}$ from the original image $I$ based on the provided security context $\kappa$. The context within which an image could be identified as authentic will typically contain some secret keys for identification of the users or the system, and the time-stamp of the image. Construction of the authentication context is out of scope of this study. Optionally, a set of requirements towards the reconstruction process can also be given at this point. Based on a provided image and context, the decoder yields a tampering map $E$, and a restored image $I^{(\mathrm{r})}$, provided that the reconstruction is possible.

The reconstruction is performed based on the communicated reference information $\mathbf{r}$, as specified in the quality descriptor q. Both $\mathbf{r}$, and $\mathbf{q}$ are communicated through an erasure channel. Due to intrinsic content authentication features, the transmitted portions of the watermark are seen as either correct, or corrupted. While the descriptor is decoded in a traditional way, decoding of the reference information is aided by exploiting the remaining authentic image content.

The reference scheme described in [10] does not use a quality descriptor, and only the reference information $\mathbf{r}$ is communicated through the image. The reference scheme can be seen as a special case of the adaptive scheme with a trivial quality descriptor specifying the same profile for all image blocks.

\section{A. Basic Notation}

Let $I$ denote the original, unprotected image and $I_{i}: i=$ $1, \ldots, N$ the $i$-th image block in the raster scan order. The reference information for individual image blocks is denoted as $r_{i}$ :

$$
\mathbf{r}=r_{1}, \ldots, r_{N}=\left\{r_{i}\right\}
$$

The length of the reference information varies for individual image block, and stems from the utilized reconstruction profiles. It is assumed that the system defines $P$ profiles. Then, the quality descriptor $q(i)$ has a form of the following mapping:

$$
q:\{1, \ldots, N\} \rightarrow\{1, \ldots, P\} .
$$

Let $h(\cdot)$ denote a hashing function, which generates a cryptographic hash from the image block content $I_{i}$, block payload $W_{i}$, block number $i$ and the security context $\kappa$. In this study, we used the MD5 algorithm, shortened to 32 bits by exclusive disjunction on neighboring pixel pairs. The resulting hash for the $i$-th image block is denoted by:

$$
H_{i}=h\left(I_{i}^{(\mathrm{ldr})}, i, W_{i}, \kappa\right) .
$$

The utilized watermark embedding scheme uses LSBS in the spatial domain. The number of bit-planes utilized for information embedding is denoted by $L$. Hence, the principal image content $I^{(\mathrm{ldr})}$, used for hash calculation, is represented by $8-L$ MSBs, i.e., $I^{(\mathrm{ldr})}=\left\lfloor I / 2^{L}\right\rfloor$. The total embedding capacity of $64 L$ bits is divided into 32 bits for the hashes, 8 bits for the quality descriptor, and the rest for the reconstruction reference.

\section{B. Methods for Image Encoding}

Operation of the encoder is illustrated in Fig. 2a. The procedure begins with a pre-processing step, which discards $L$ least significant bit-planes in order to limit the number of unique colors in the image. This step is followed by blockwise computation of the DCT spectrum. We consider a fixed block size of $8 \times 8$ px.

The following reconstruction reference generation step is controlled by the quality descriptor. The encoder calculates the reconstruction profile mapping to optimize the reconstruction quality, given prospective operation constraints or requirements. The details of this step are described in detail in Section V. The reference information is obtained by encoding individual DCT coefficients. A reconstruction profile defines the total number of bits per block $b$, the precision of individual coefficients, and the quantization code-books. The latter two can be easily adapted to various content characteristics. In our study, we use uniform quantization for the DC coefficients, and Lloyd-Max code-book for AC coefficients. The precision of the coefficients, represented by means of a $8 \times 8$ matrix:

$$
\mathbf{V}=\left[v_{i, j}\right]_{8 \times 8}: v_{i, j} \in\{0,1, \ldots, 8\} \text {. }
$$




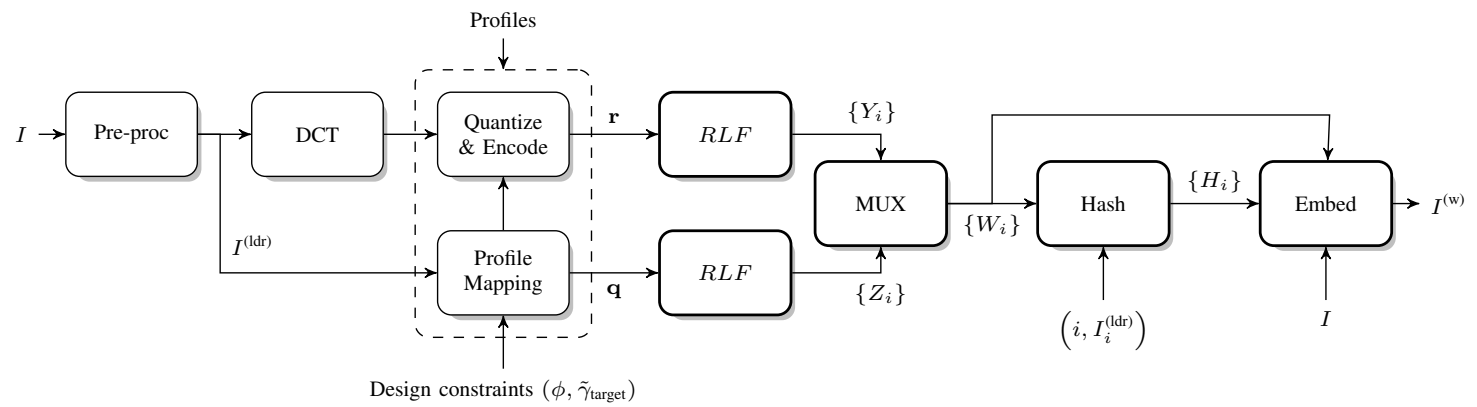

(a) Operation of the encoder

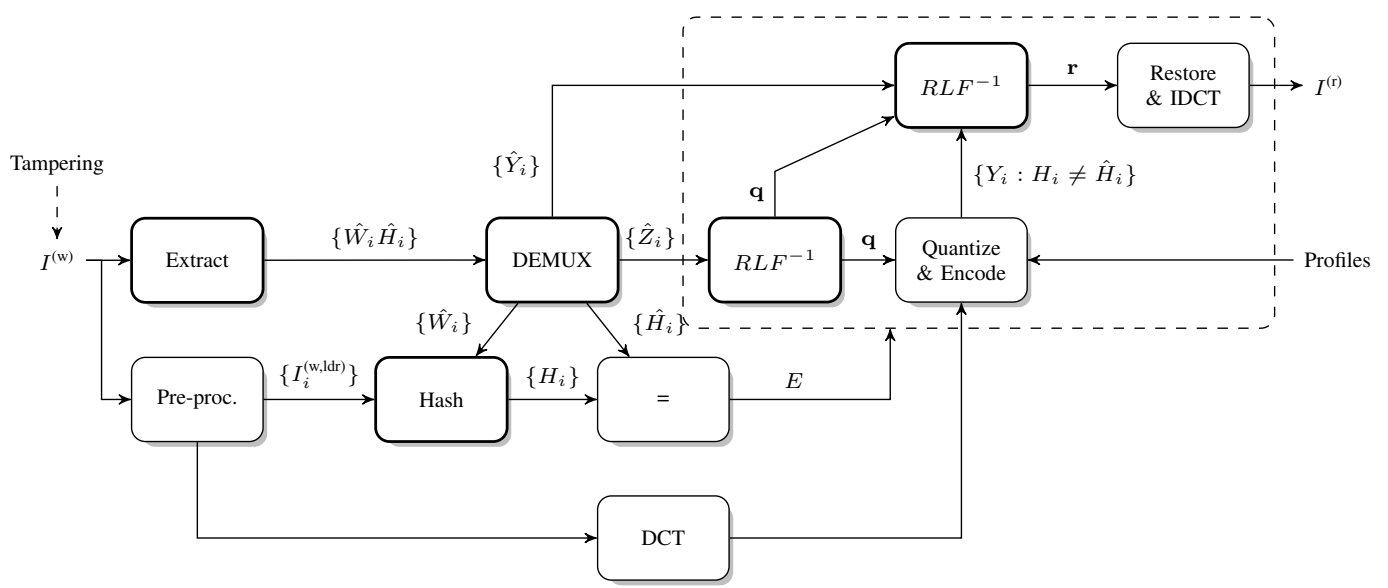

(b) Operation of the decoder

Fig. 2: Operation of the adaptive self-embedding scheme; $I$ - cover image, $I^{(\mathrm{w})}$ - protected, watermarked image, $I^{(\mathrm{r})}$ - recovered, authenticated image, $E$ - tampering map, q - quality descriptor; steps directly controlled by the security context $\kappa$ are marked by thick borders.

is chosen by solving the following optimization problem:

$$
\begin{aligned}
\min & \theta(\mathbf{V}), \\
\text { s.t. } & \forall_{(i, j) \in\{1, \ldots, 8\}^{2}} \quad 0 \leq v_{i, j} \leq 8, \\
& \forall_{(i, j) \in\{1, \ldots, 8\}^{2}} \quad v_{i, j} \in \mathbf{Z}, \\
& \sum_{(i, j) \in\{1, \ldots, 8\}^{2}} v_{i, j}=b .
\end{aligned}
$$

where $\theta(\mathbf{V})$ is a cost function, representing the average distortion for a specific precision allocation V. Condition (5b) ensures that the precision is in the range $0-8$ bits, and $(5 \mathrm{c})$ constrains the solution to integers only. The payload budget is guaranteed by $(5 \mathrm{~d})$.

Both the quality descriptor, and the reconstruction reference are communicated to the decoder through an erasure channel. The communication model in the former case corresponds to a generic erasure transmission [15], while in the latter to the self-recovery transmission [10]. Both $\mathbf{r}$ and $\mathbf{q}$ are divided into constant-length symbols (after zero-padding, if necessary):

$$
\begin{gathered}
\mathbf{r} \rightarrow X_{1}, \ldots, X_{\left\lceil\lambda_{\mathrm{r}} N\right\rceil}, \\
\mathbf{q} \rightarrow Q_{1}, \ldots, Q_{\left\lceil\lambda_{\mathrm{q}} N\right\rceil},
\end{gathered}
$$

where $\lambda_{\mathrm{r}}$ and $\lambda_{\mathrm{q}}$ are the code rates for the reconstruction reference and the quality descriptor, respectively. The reference rates stem from the rate of the information about a particular block to its entitled embedding capacity, e.g., the reference rate for $i$-th block:

$$
\lambda(i)=\frac{b(i)}{64 L-40} .
$$

In traditional self-embedding with uniform reconstruction quality, the code rate $\lambda_{\mathrm{r}}$ is equal to the global reference rate $\lambda(i) \equiv \lambda$, which is a system parameter directly used for controlling the reconstruction quality. In adaptive self embedding, the reference rates might be different for various image blocks, i.e., $\lambda(i)$, and $\lambda_{\mathrm{r}}$ is calculated as an average rate for all image blocks:

$$
\lambda_{\mathrm{r}}=\frac{1}{N} \sum_{i=1}^{N} \lambda(i) .
$$

Due to the fact that the proposed approach can exploit the remaining authentic image content, the reference rates can safely be $\geq 1$, and hence typically $\lambda_{\mathrm{r}}>1$. The quality descriptor is communicated through a traditional erasure channel, and the corresponding code rate $\lambda_{\mathrm{q}}<1$ needs to be selected to match the target tampering rate. In this study, we consider $\lambda_{\mathrm{q}}=1 / 2$, which allows for the maximum tampering rate of 0.5 - the same as for the lowest considered quality level.

The resulting symbols are then encoded with a fountain code to produce exactly $N$ same-length output symbols, for each 
image block:

$$
\begin{aligned}
& X_{1}, \ldots, X_{\lambda_{\mathrm{r}} N} \stackrel{R L F}{\longrightarrow} Y_{1}, \ldots, Y_{N}, \\
& Q_{1}, \ldots, Q_{\lambda_{\mathrm{q}} N} \stackrel{R L F}{\longrightarrow} Z_{1}, \ldots, Z_{N} .
\end{aligned}
$$

The utilized fountain code is the random linear fountain (RLF) [16]. It generates output symbols by combining pseudorandomly selected input symbols using exclusive disjunction. The input symbols are selected independently, with probability 0.5 . A decoder will be able to decode the communicated message from arbitrarily chosen symbols, provided that the number of available symbols is sufficient. Due to elimination of the knowledge about authentic image fragments, sparse fountain codes are not well suited for the application at hand [10].

The resulting symbols $Y_{i}, Z_{i}$ are then pseudo-randomly combined by a multiplexer (MUX) to yield the embedding payload for the $i$-th image block, $W_{i}$. The multiplexer operates by pseudo-randomly selecting and shuffling the bits from the input streams. This step could be simplified by simple concatenation without any performance penalty, but adoption of the multiplexer introduces additional randomization of the reconstruction process.

The watermark payload is then fed along with image block content $I_{i}^{(\mathrm{ldr})}$, image block location $i$, and the security context $\kappa$ to the hashing function $h(\cdot)$. The resulting hashes $H_{i}$ are embedded along with $W_{i}$ into appropriate image blocks. This concludes the operation of the encoder.

\section{Methods for Image Decoding}

Operation of the adaptive self-embedding decoder is illustrated in Fig. 2b. The process starts with watermark extraction, which yields the extracted hashes $\hat{H}_{i}$, and watermark payload $\hat{W}_{i}$, potentially different from the embedded original information. In the next step, the decoder recalculates the hashes:

$$
H_{i}=h\left(I_{i}^{(\mathrm{w}, \mathrm{ldr})}, i, \hat{W}_{i}, \kappa\right),
$$

by repeating the necessary steps of the encoder. Comparison of $H_{i}$ and $\hat{H}_{i}$ gives the tampering (erasure) map $E$, necessary for fountain decoding.

The remaining authentic $\hat{W}_{i}$ are demultiplexed (DEMUX) to obtain $\left\{\hat{Y}_{i}: H_{i}=\hat{H}_{i}\right\}$, and $\left\{\hat{Z}_{i}: H_{i}=\hat{H}_{i}\right\}$. Provided that the number of authentic symbols is sufficient for successful decoding of both streams, the decoder will be able to restore the original image's content. Otherwise, the operation terminates, and the decoder yields only the tampering map $E$.

At first, the decoder recovers the quality descriptor q. Based on the assigned quality profiles, it then regenerates the reference information of authentic image regions. The dependencies on the regenerated reference symbols are then removed from the previously extracted $\left\{\hat{Y}_{i}: H_{i}=\hat{H}_{i}\right\}$. The second fountain decoding yields the reference information of the tampered image blocks. The last step is to restore their principal content.

\section{Communication of the Quality Descriptor}

The quality descriptor is communicated to the decoder through the same channel as the reconstruction reference. The rate of the fountain code should be chosen accordingly to the anticipated maximal tampering rate. It is necessary to decode the whole quality descriptor. The maximal tampering rate $\tilde{\gamma}$ which allows for its successful retrieval is asymptotically:

$$
\tilde{\gamma}_{\mathrm{q}}=1-\lambda_{\mathrm{q}} .
$$

Further improvement of $\tilde{\gamma}_{\mathrm{q}}$ could be obtained by employing source coding to compress the descriptor prior to channel coding. This issue is out of scope of this study.

\section{Theoretical AnAlysis of CONTENT AdAPtivity}

For an ideal fountain code successful reconstruction is possible, if the number of available watermark symbols is greater or equal to the number of necessary reference symbols:

$$
\begin{gathered}
1-\tilde{\gamma} \geq \lambda_{\mathrm{r}} \rho(\tilde{\gamma} \mid q, E), \\
\rho(\tilde{\gamma} \mid q, E): \mathbf{R}^{+} \times[0,1] \rightarrow[0,1] .
\end{gathered}
$$

where $\rho(\tilde{\gamma} \mid q, E)$ is the reconstruction demand, i.e., the fraction of the reference stream which needs to be decoded from the extractable watermark information. The growth of the reconstruction demand depends on the reference rates specified by the quality descriptor $\mathbf{q}$, and on the selection of blocks for modification $E$. In order to derive a representative performance measure, it is beneficial to consider the expected behavior of the reconstruction demand, estimated as the average over all possible tampering patterns:

$$
\rho(\tilde{\gamma} \mid q)=\frac{1}{\# \Omega} \sum_{E \in \Omega} \rho(\tilde{\gamma} \mid q, E)
$$

where $\Omega$ denotes the set of all possible tampering maps, and $\# \Omega$ denotes the number of elements in $\Omega$.

Ideally, the reconstruction demand should be proportional to the tampering rate:

$$
\rho(\tilde{\gamma} \mid q, E)=\tilde{\gamma},
$$

regardless of the tampering map $E$, and quality descriptor $\mathbf{q}$. For traditional, uniform-quality self-embedding, this can be ensured by choosing a single, natural reference rate $\lambda(i) \equiv$ $\lambda_{\mathrm{r}} \in \mathbf{N}^{+}$. In such case, the reconstruction is possible up to the optimistic success bound [10]:

$$
\begin{aligned}
1-\tilde{\gamma} & \geq \lambda_{\mathrm{r}} \tilde{\gamma}, \\
\tilde{\gamma} & \leq \frac{1}{\lambda_{\mathrm{r}}+1} .
\end{aligned}
$$

For further analysis of content adaptivity, we consider a more convenient form of the success condition (12), where equality defines the reconstruction success bound:

$$
\rho(\tilde{\gamma} \mid q, E)=\lambda_{\mathrm{r}}^{-1}(1-\tilde{\gamma}) .
$$

The right hand side represents the fraction of the available watermark symbols, normalized with respect to the total number of reference symbols. It is always a linear function with slope inversely proportional to the reference rate $\lambda_{\mathrm{r}}$. Fig. 3 shows 


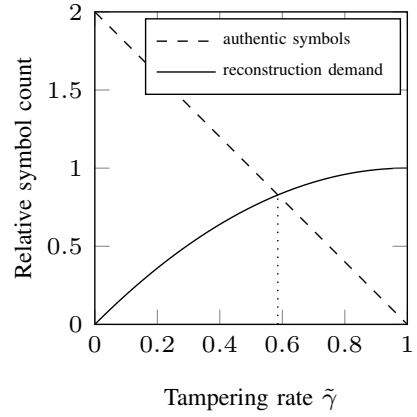

(a) $\lambda=0.5, \tilde{\gamma}_{\max }=0.586$

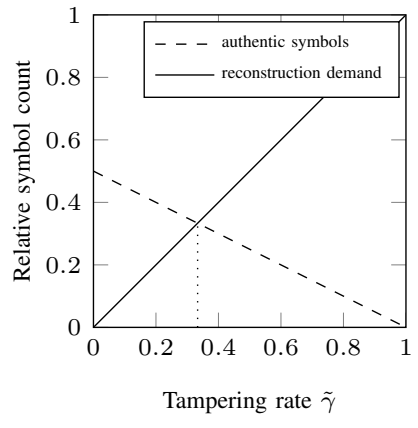

(b) $\lambda=2, \tilde{\gamma}_{\max }=0.333$

Fig. 3: Graphical interpretation of the success bound.

a graphical representation of (15) for two example uniformquality configurations: $\lambda_{\mathrm{r}}=0.5$, and $\lambda_{\mathrm{r}}=2$. The maximal tampering rate corresponds to the intersection between the two functions.

In adaptive schemes the defined reconstruction profiles are characterized by various reference rates. However, in general, there might exist multiple profiles with the same reference rates, i.e., $\lambda_{i}=\lambda_{j}: i \neq j$, but differentiated by other profile characteristics, e.g., quantization code-book, or coefficient precision assignment. Hence, the quality descriptor implicitly determines the mapping $\lambda(i)$ :

$$
\lambda:\{1, \ldots, N\} \rightarrow\left\{\lambda_{1}, \ldots, \lambda_{S^{\prime}}\right\},
$$

where $S^{\prime}$ denotes the number of possible unique reference rates. This mapping is fundamental in the analysis of the reconstruction success bounds in adaptive self-embedding systems. Let:

$$
\Lambda=\left[\lambda_{1}, \ldots, \lambda_{S}\right]: \lambda_{1}>\ldots>\lambda_{S}
$$

be a vector of reference rates used in a given quality descriptor. We focus on natural rates, i.e., $\forall_{i \in\{1, \ldots, N\}} \lambda(i) \in \mathbf{N}^{+}$, which guarantees that for each of these rates alone, the scheme reaches the optimistic success bound (14b).

The occurrence frequency for each reference rate can be represented by a weight vector w:

$$
\mathbf{w}=\left[w_{1}, w_{2}, \ldots, w_{S}\right]: w_{s} \in(0 ; 1) \wedge \sum_{s=1}^{S} w_{s}=1,
$$

with $S-1$ degrees of freedom. Hence:

$$
w_{S}=1-\sum_{s=1}^{S-1} w_{s} \text {. }
$$

\section{A. Reconstruction Demand Analysis}

Incorporation of multiple reconstruction profiles with various reference rates impacts on the reconstruction success bounds. Depending on the selection of maliciously tampered blocks, the system tolerates various tampering rates. The problem can be theoretically analyzed by determining the behavior of the reconstruction demand. Two representative cases can be distinguished: a pessimistic success bound $\tilde{\gamma}_{\text {min }}$, and an average success bound $\tilde{\gamma}_{\text {ave }}$.

1) Pessimistic Success Bound: $\tilde{\gamma}_{\min }$ is the minimum tampering rate that can render the reconstruction impossible. It is applicable when the reconstruction demand assumes the steepest possible growth, i.e., when the blocks are tampered with decreasing order of reference rates $\lambda(i)$. This scenario corresponds to a realistic assumption that the most important, and hence tampering-prone, content is likely to be assigned higher values of $\lambda$. Within these implicitly defined content importance levels no particular secondary importance is assumed. During simulations, image blocks with the same importance are chosen randomly. Such modifications are referred to as the pessimistic tampering.

For pessimistic tampering, the reconstruction demand becomes a piecewise-linear function with slopes dependent on both the reference rates, and their weights. Let $w_{s}^{+}$denote the cumulative weight for the $s$-th reference rate:

$$
w_{s}^{+}= \begin{cases}0, & \text { for } s=0, \\ \sum_{i=1}^{s} w_{i}, & \text { for } s=1, \ldots, S .\end{cases}
$$

Then, given a set of reference rates $\Lambda$ with weights $\mathbf{w}$, the reconstruction demand is:

$$
\rho(\tilde{\gamma} \mid \Lambda, \mathbf{w})= \begin{cases}\alpha_{1} \tilde{\gamma}+\beta_{1}, & \text { for } \tilde{\gamma} \in\left(0, w_{1}^{+}\right], \\ \cdots & \text { for } \tilde{\gamma} \in\left(w_{s-1}^{+}, w_{s}^{+}\right], \\ \alpha_{s} \tilde{\gamma}+\beta_{s}, & \\ \cdots & \text { for } \tilde{\gamma} \in\left(w_{S-1}^{+}, 1\right] .\end{cases}
$$

where the slopes $\alpha_{s}$ can be calculated as:

$$
\alpha_{s}=\lambda_{s} / \sum_{i=1}^{S} w_{i} \lambda_{i}
$$

and the y-intercepts $\beta_{s}$ for individual components are chosen to ensure function continuity:

$$
\beta_{s}: \bigvee_{s \in\{1, \ldots, S\}} \rho\left(w_{k-1}^{+} \mid \Lambda, \mathbf{w}\right)=\lim _{\tilde{\gamma} \rightarrow\left(w_{k-1}^{+}\right)^{+}} \rho(\tilde{\gamma} \mid \Lambda, \mathbf{w}) .
$$

For the currently considered scenario, the y-intercepts $\beta_{s}$ can be calculated as:

$$
\beta_{k}= \begin{cases}0, & \text { for } k=1, \\ \left(\alpha_{k-1}-\alpha_{k}\right) w_{k-1}^{+}+\beta_{k-1}, & \text { otherwise. }\end{cases}
$$

Fig. 4 shows the reconstruction demand $\rho$ for a randomly generated quality descriptor with $\Lambda=[3,2,1]$ and $\mathbf{w}=[0.1429,0.2857,0.5714]$. (a) shows the piecewiselinear character of the resulting function, and the slopes of its successive components. The theoretically expected character is verified experimentally by performing random reconstruction attempts after applying pessimistic tampering. Experimental results are shown in (b). Individual reconstruction attempts are marked with circles and crosses, for successful and unsuccessful reconstruction, respectively. The resulting success bound $\tilde{\gamma}_{\min }=0.2864$.

It is also possible to distinguish a dual, optimistic success bound, observed when image blocks with minimal impact on $\rho$ are tampered first. Such tampering has no reasonable justification in prospective forgery scenarios, and is therefore not considered. 


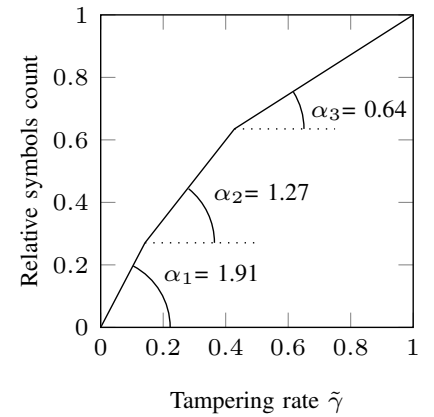

(a) Piecewise-linear character of $\rho$

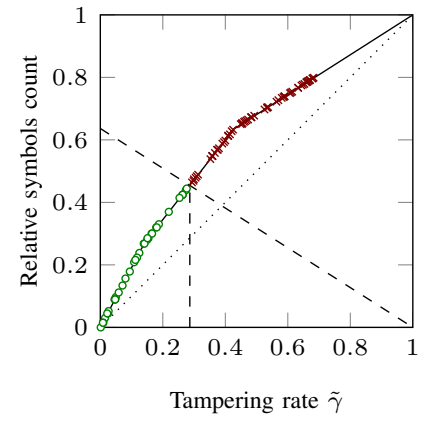

(b) Experimental validation
Fig. 4: Reconstruction demand for pessimistic tampering.

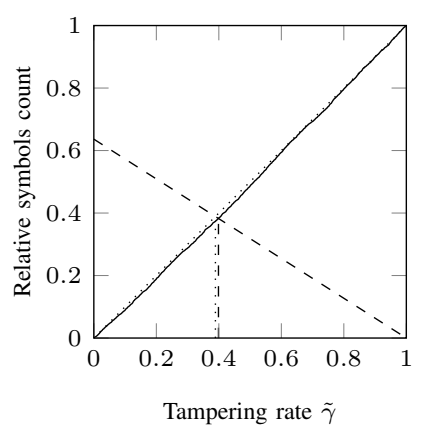

(a) Example realization

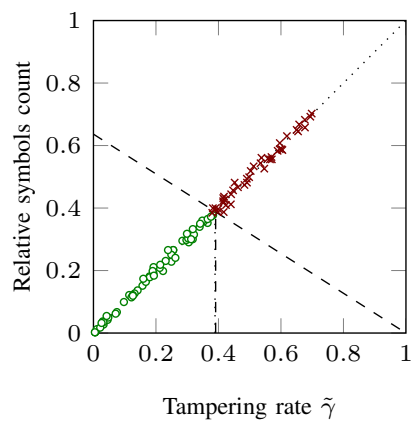

(b) Experimental validation

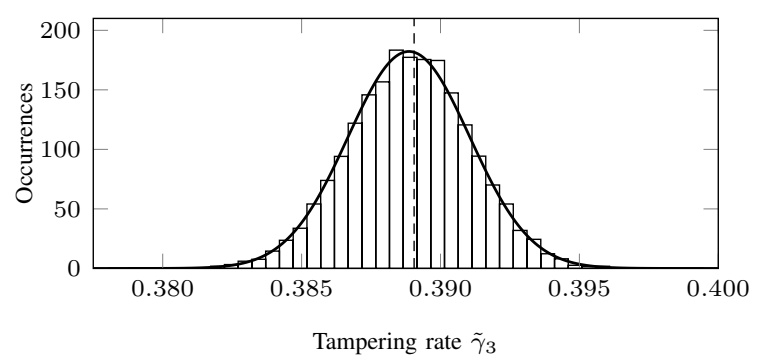

(c) Histogram of actual bounds for 10,000 random realizations

Fig. 5: Linear growth of the reconstruction demand $\rho$, and the resulting success bound for $\Lambda=[3,2,1]$ and $\mathbf{w}=$ $[0.1429,0.2857,0.5714]$.

2) Average Success Bound: Oblivious tampering randomly selects image blocks for modification. For each individual realization of the tampering process, the achievable success bound will be slightly different, depending on the quality settings of the tampered blocks, and the resulting curve of the reconstruction demand. The average tampering rate represents an estimate of the expected value of the tampering rate bounds.

It can be shown, that for oblivious tampering, the reconstruction demand has stochastic character with oscillations around the expected linear growth. An example realization of the process is shown in Fig. 5a with a solid line. The average linear growth is marked with a dotted line. Since the analysis is relative to the total number of reference symbols, the slope of the expected growth is always 1 , i.e., $\rho(\tilde{\gamma} \mid \Lambda, \mathbf{w})=\tilde{\gamma}$. Hence, the average success bound can be calculated as:

$$
\tilde{\gamma}_{\text {ave }}=\frac{1}{1+\lambda_{\mathrm{r}}} \text {. }
$$

where $\lambda_{\mathrm{r}}$ is the average reference rate.

Fig. 5b shows the results of experimental validation of the described behavior. The illustrated example is the same as for the pessimistic tampering, i.e., $\Lambda=[3,2,1]$ and $\mathbf{w}=[0.1429,0.2857,0.5714]$. The average success bound is $\tilde{\gamma}_{\text {ave }}=0.3889$. Since individual realizations oscillate around the linear growth, the actual bound oscillates around $\tilde{\gamma}_{\text {ave }}$. (c) shows a histogram of the bounds obtained for individual realizations of the process, along with a fitted Gaussian pdf. The sample mean from 10,000 simulated realizations is 0.3888 . According to the $3 \sigma$ rule, $99.7 \%$ of the observed values fall in the range $[0.3823 ; 0.3954]$.

\section{B. Impact of Multiple Quality Profiles}

In this section, we investigate the impact of incorporating multiple reconstruction profiles on the success bounds $\tilde{\gamma}_{\text {min }}$ and $\tilde{\gamma}_{\text {ave }}$. Our analysis provides an insight into the improvement, in terms of the tampering rate, that can be achieved by introducing quality levels with lower restoration fidelity.

For a setting with two profiles, the corresponding weight vector has one degree of freedom:

$$
\mathbf{w}=[w, 1-w],
$$

and the parameter $w$ alone is sufficient for controlling the proportion between the high-quality and low-quality blocks. The reference rates $\lambda_{1}$ and $\lambda_{2}$ control the asymptotic tampering rates for $w \rightarrow 0$, and $w \rightarrow 1$. It can be shown that there is a threshold value of $w$, below which there is no improvement in terms of the pessimistic tampering rate $\tilde{\gamma}_{\text {min }}$.

Proposition 1. For a two-profile configuration of an adaptive self-embedding scheme with reference rates $\lambda_{1}>\lambda_{2}$, the pessimistic tampering rate bound for weights $w \in\left(\frac{1}{1+\lambda_{1}}, 1\right]$ is constant and dependent only on the higher reference rate $\lambda_{1}$.

Proof. A two-profile configuration is described by reference rates $\Lambda=\left[\lambda_{1}, \lambda_{2}\right]$, and weights $\mathbf{w}=[w, 1-w]$. From (18)(21), for any $w \in[0,1]$ the reconstruction demand $\rho(\tilde{\gamma} \mid \Lambda, \mathbf{w})$ can be rewritten for a pessimistic tampering pattern as:

$$
\rho(\tilde{\gamma} \mid \Lambda, \mathbf{w})= \begin{cases}\frac{\lambda_{1}}{\lambda_{\text {ave }}} \tilde{\gamma}, & \text { for } \tilde{\gamma} \in(0, w], \\ \frac{\lambda_{2}}{\lambda_{\text {ave }}} \tilde{\gamma}+\left(\frac{\lambda_{1}}{\lambda_{\text {ave }}}-\frac{\lambda_{2}}{\lambda_{\text {ave }}}\right) w, & \text { for } \tilde{\gamma} \in(w, 1] .\end{cases}
$$

For the first component, the solution to (15) dependents only on $\lambda_{1}$ :

$$
\begin{aligned}
\frac{\lambda_{1}}{\lambda_{\text {ave }}} \tilde{\gamma}_{\text {min }} & =\frac{1}{\lambda_{\text {ave }}}\left(1-\tilde{\gamma}_{\text {min }}\right), \\
\tilde{\gamma}_{\text {min }} & =\frac{1}{1+\lambda_{1}} .
\end{aligned}
$$

The first component determines the reconstruction success bound as long as the left hand side of (15) is greater than the right hand side at the boundary between the reconstruction demand components, i.e., $\tilde{\gamma}=w$ :

$$
\rho(w \mid \lambda, \mathbf{w})>(1-w) / \lambda_{\text {ave }},
$$




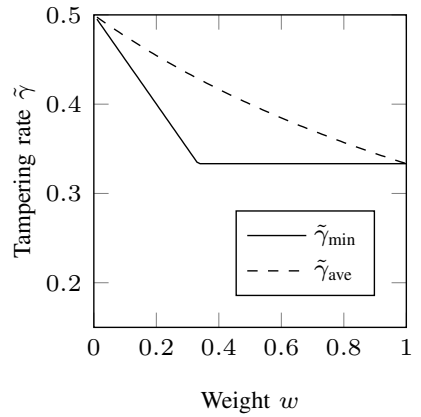

(a) Two profiles, $\lambda_{1}=2, \lambda_{2}=1$

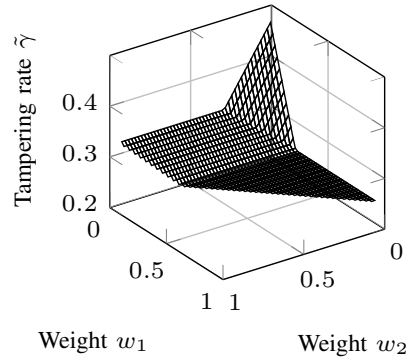

(c) Pessimistic bound, $\Lambda=[3,2,1]$

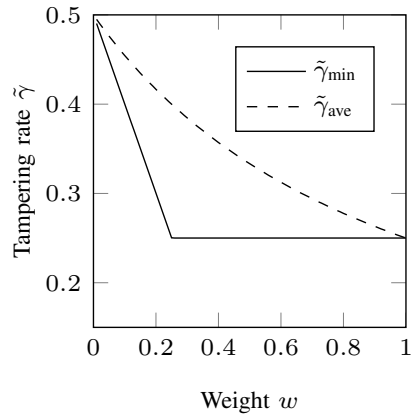

(b) Two profiles, $\lambda_{1}=3, \lambda_{2}=1$

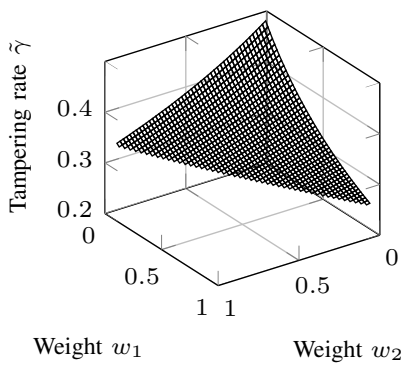

(d) Average bound, $\Lambda=[3,2,1]$

Fig. 6: Impact of multiple quality profiles on the pessimistic, and the average success bounds for configurations with two, and three quality profiles.

$$
w>\frac{1}{1+\lambda_{1}}
$$

It is also possible to derive an analytical expression for the reconstruction demand for the remaining range $w \in\left[0, \frac{1}{1+\lambda_{1}}\right)$ :

$$
\begin{aligned}
\frac{\lambda_{1}}{\lambda_{\text {ave }}} \tilde{\gamma}_{\text {min }}+\frac{\lambda_{1}-\lambda_{2}}{\lambda_{\text {ave }}} w & =\frac{1}{\lambda_{\text {ave }}}(1-\tilde{\gamma}), \\
\tilde{\gamma}_{\text {min }} & =\frac{1+\left(\lambda_{2}-\lambda_{1}\right) w}{\lambda_{2}+1} .
\end{aligned}
$$

Fig. 6ab show both the pessimistic and the average success bounds for $\Lambda=[2,1]$, and $\Lambda=[3,1]$. It is clearly visible that while the average bound systematically improves, the pessimistic bound remains unchanged until most of the blocks are assigned the lower-quality profile. Analogous behavior is also observed when more reconstruction profiles are used. Fig. 6cd show the pessimistic, and the average success bounds for a three-profile configuration of $\Lambda=[3,2,1]$ with two degrees of freedom $\mathbf{w}=\left[w_{1}, w_{2}, 1-w_{1}-w_{2}\right]$.

\section{Quality Descriptor Design}

There are two main reasons, which motivate the use of quality descriptors. Firstly, they allow for additional improvement of the achievable reconstruction quality. A set of reconstruction profiles can be designed with the use of various reconstruction strategies, suited for image blocks with different content. For instance, coefficient quantization code-books, or their precision might change for blocks with distinct texture levels.

Secondly, the quality descriptor allows for enforcing the desired reconstruction quality for selected image blocks. The

quality of the remaining background content can then be adjusted to meet the requirements towards the tampering rate.

In general, the complete quality descriptor calculation procedure involves two phases: profile mapping, and descriptor design. The former selects the profiles which yield the highest reconstruction quality. The latter gradually reduces reconstruction quality of individual blocks in order to meet the target tampering rate constraint.

\section{A. Definition of Requirements}

The considered set of requirements for the quality descriptor design procedure includes an importance map $\phi$, the requested target tampering rate, success bound type (i.e., pessimistic, or average), a set of guard quality profiles $\mathbf{G}$, and other minor, implementation-specific parameters.

The importance map $\phi(i)$ :

$$
\phi:\{1, \ldots, N\} \rightarrow\{0,1\}
$$

indicates which blocks should be reconstructed with maximal possible quality $(\phi(i)=1)$, and which can be adapted to satisfy other design requirements $(\phi(i)=0)$. Further extension of the possible importance levels, e.g., to support exclusion of image blocks from reconstruction, is straightforward, and will not be discussed in this study.

All image blocks are initially assigned their optimal reconstruction profiles during a profile mapping phase. For important image blocks, i.e., $\{i: \phi(i)=1\}$, this assignment is final. The assignment for the remaining unimportant blocks is systematically demoted during successive iterations of the descriptor design phase. The degradation follows until the design objective, the requested target tampering rate $\tilde{\gamma}_{\text {target }}$, is met.

In order to prevent excessive degradation, the design procedure uses a set of guard quality profiles $\mathbf{G}$, below which an individual block cannot be degraded. This concept is particularly useful if the given set of reconstruction profiles distinguishes multiple block texture levels. It prevents incorrect assignment to a wrong block type.

\section{B. Profile Mapping Procedure}

The goal of profile mapping is twofold. Firstly, it provides an initial quality descriptor $q_{0}$, which optimizes the reconstruction quality by choosing the best possible profile:

$$
q_{0}(i): \bigvee_{i=\{1, \ldots, N\}} q_{0}(i)=\underset{p \in\{1, \ldots, P\}}{\operatorname{argmin}} \operatorname{MSE}\left(I_{i}, I_{i}^{(\mathrm{ref} \mid p)}\right) .
$$

where $I_{i}^{(\text {ref } \mid p)}$ denotes an approximate version of the $i$-th block, recovered from its reference information using the $p$-th reconstruction profile.

Secondly, the mapping procedure yields a distortion map:

$$
d(i, p):\{1, \ldots, N\} \times\{1, \ldots, P\} \rightarrow \operatorname{MSE}\left(I_{i}, I_{i}^{(\operatorname{ref} \mid p)}\right)
$$

needed in the following descriptor design phase. For the sake of computation efficiency, the distortion map may include only 


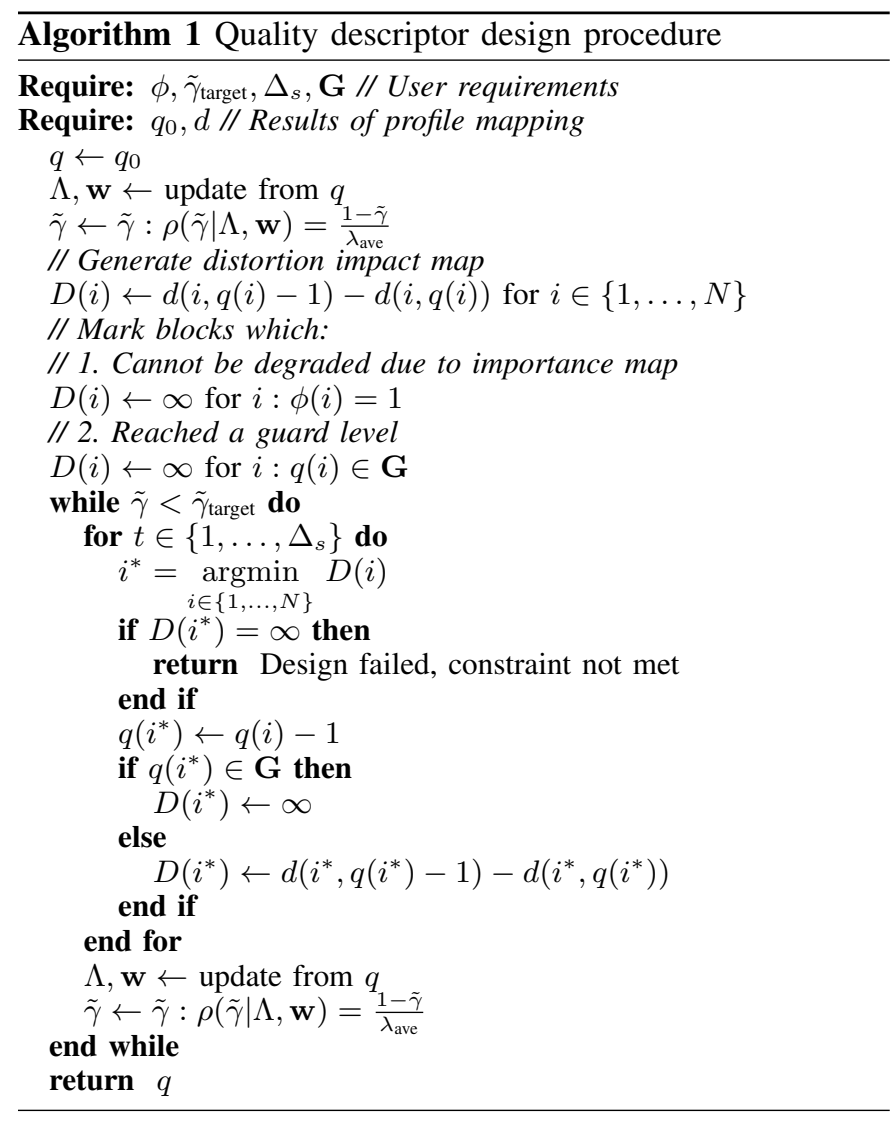

the allowed profiles for each image block. If the given set of reconstruction profiles is based on a single block texture level, the profile mapping becomes trivially $\vee_{i} q(i)=P=S$, yet it is still necessary to calculate the distortion map $d(i, p)$.

\section{Descriptor Design Procedure}

The descriptor design procedure relies on the initial descriptor, and the distortion map obtained from the preceding profile mapping phase. The general idea is to perform iterative degradation of successive image blocks. In each step, the current tampering rate is calculated and compared against the target design objective. Image blocks for degradation are selected in the order of increasing degradation impact $D$ :

$$
D(i) \leftarrow d(i, q(i)-1)-d(i, q(i)),
$$

while respecting the content importance provided by $\phi$, and the guard levels $\mathbf{G}$.

The degradation is performed in batches of $\Delta_{s}$ image blocks. By increasing this parameter it is possible to speed up the process, at the cost of a slight delay in finding that the target tampering rate has already been reached. The complete procedure is presented as Algorithm 1.

\section{EXPERIMENTAL EVALUATION}

In the performed experimental evaluation, we focus on two principal aspects. Firstly, we validate the descriptor design procedure to confirm that the target tampering rates can actually be reached. Secondly, we assess the reconstruction
TABLE I: The considered reconstruction profiles.

\begin{tabular}{lrrrrrrrr}
\hline Profile no. & 1 & 2 & 3 & 4 & 5 & 6 & 7 & 8 \\
\hline$\lambda$ & 0 & 1 & 1 & 2 & 3 & 1 & 2 & 3 \\
Block texture & - & L & M & M & M & H & H & H \\
Guard & - & + & + & - & - & + & - & - \\
\hline
\end{tabular}

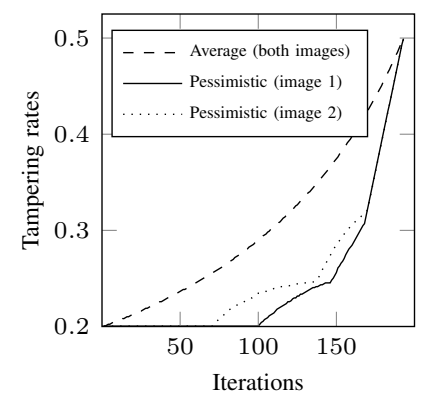

(a) Tampering rates for successive iterations of the design process

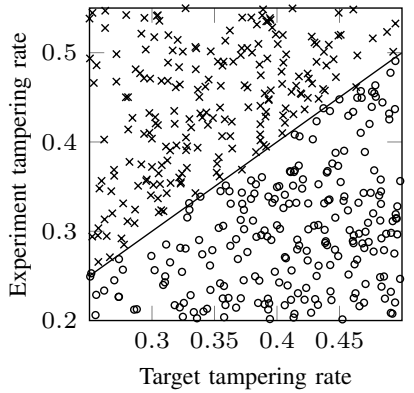

(b) Design objective validation
Fig. 7: Intermediate tampering rates and validation result of the descriptor design process.

efficiency, i.e., ability to trade-off the reconstruction quality for the tampering rate.

All of the described experiments were performed using the adaptive self-embedding scheme described in Section III. Its performance is compared to the reference scheme from [10], which features uniform-quality restoration. Both schemes are configured to use two least significant bit-planes for data embedding, i.e., $L=2$. Hence, the embedding capacity is 128 bits per block, from which 32 bits are used for embedding content hashes. The reference scheme uses the remaining 96 bits for embedding the reconstruction reference.

The adaptive scheme needs to communicate the quality descriptor, and uses 8 bits for this purpose. Hence, the reference symbol length is 88 bits. The descriptor is represented with 4bit depth, and is encoded with a fountain code of rate $\lambda_{\mathrm{q}}=\frac{1}{2}$. The utilized reconstruction profiles (Table I) use reference rates between 1 and 3, and are intended for low (L), medium $(\mathrm{M})$, and high $(\mathrm{H})$ texture blocks.

Image blocks are classified to either texture category based on the standard deviation of their pixels:

$$
\begin{cases}\text { low-texture block, } & \text { if } \sigma_{i} \leq 5, \\ \text { medium-texture block, } & \text { if } 5<\sigma_{i} \leq 20, \\ \text { high-texture block, } & \text { if } \sigma_{i}>20\end{cases}
$$

where $\sigma_{i}$ denotes the standard deviation for the $i$-th image block. The thresholds were selected by visual inspection of the resulting block sets.

\section{A. Descriptor Design Validation}

The purpose of this experiment is to validate the achievable success bounds for the designed quality descriptors. The encoder prepares a number of quality descriptors for a range of considered target tampering rates $\tilde{\gamma}_{\text {target }} \in[0.25,0.5]$. Fig. 7a shows how the achievable tampering rates change with successive iterations of the procedure. The average tampering 


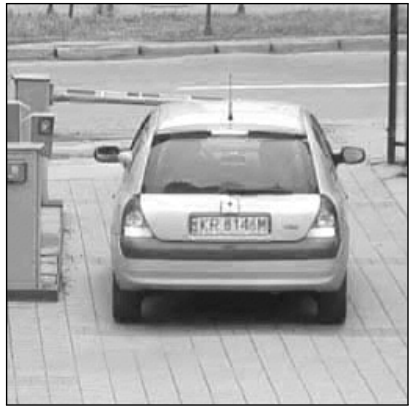

(a) Reference, $32.2 \mathrm{~dB}$

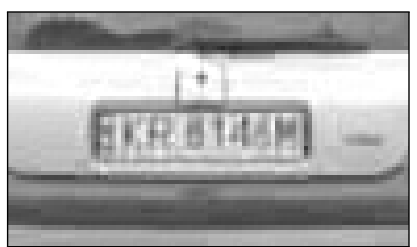

(c) Close-up of (a)

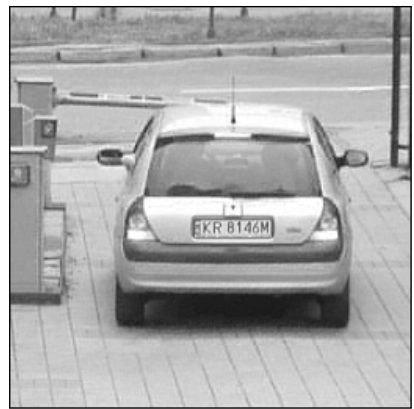

(b) Adaptive, $33.0 \mathrm{~dB}$

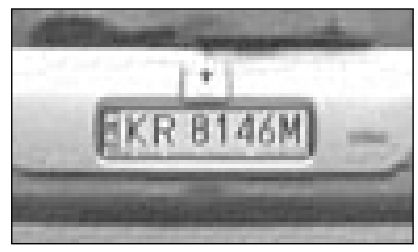

(d) Close-up of (b)
Fig. 9: Comparison of reference images for the uniformquality, and the adaptive schemes for the same tampering rate bound.

rate behaves identically, regardless of the image content. Consistently with theoretical analysis, the pessimistic tampering rate starts to increase after a certain delay, and its growth is content-dependent. Two example graphs are shown in Fig. 7a.

The reconstruction success chart for randomly drawn tampering rates is shown in Fig. 7b. Success and failure cases are marked with circles and crosses, respectively. It can be observed, that the scheme allows for successful reconstruction up to the desired target tampering rate. The experiment was repeated both for pessimistic, and for oblivious tampering. The resulting quality descriptors for selected tampering rates are shown in Fig. 8. The figure illustrates example images, and their corresponding descriptors. The top and middle rows correspond to uniform degradation, while the bottom row uses an importance map to guarantee high-quality reconstruction for the license number plate. As a result, the license number remains perfectly clear, regardless of the desired tampering rate, and the fidelity of the remaining regions.

Fig. 9 shows the prospective reconstruction result, i.e., the reference images, for both the adaptive, and the reference schemes. Both schemes allow for reconstruction up to the same tampering rate. While the reference scheme has higher effective payload (96 vs. 88 bits), it does not allow for clear reconstruction of the license number plate.

\section{B. Reconstruction Efficiency}

The goal of this experiment is to compare the achievable reconstruction efficiency with the uniform-quality reference scheme. For this purpose, we evaluate the reconstruction quality for successive target tampering rate bounds. The reference scheme is evaluated in three configurations, $\lambda=1,2$, and 3 . The adaptive scheme uses the same reference rates, but allows for assigning them to individual image blocks. This introduces an additional degree of freedom in controlling the trade-off between the reconstruction quality and the tampering rate. In fact, arbitrary tampering rates from the range:

$$
\tilde{\gamma} \in\left(\frac{1}{1+\lambda_{S}} ; \frac{1}{1+\lambda_{1}}\right)
$$

can be obtained with an appropriate profile assignment.

Due to additional capacity required for communication of the quality descriptor, the adaptive scheme uses lower effective payload for the reconstruction reference. This might lead to slight degradation of the reconstruction quality, especially at the end-points of the efficiency characteristics. At the same time, the availability of dedicated profiles, and the flexibility in their assignment allows for mitigation of the fidelity loss, or even improvement of the achievable quality. This behavior can be observed in Fig. 10, which shows example trade-off characteristics.

Fig. 10ab show the efficiency for two different images. The achievable improvement of the reconstruction efficiency is highly dependent on the image content. For images with flat, nearly solid areas (e.g., 4767 in Fig. 8a) the efficiency can be improved for both the pessimistic, and oblivious tampering. For the former, the efficiency drops rapidly at the beginning, i.e., near $\tilde{\gamma}=0.25$. This result is fully consistent with the theoretical analysis. For highly textured images (e.g., 9011 in Fig. 8a), pessimistic tampering always leads to worse reconstruction efficiency, and the improvement can be observed only for the average success bound.

Fig. 10c is an average over 50 natural images from the BOWS2 data-set [17]. It can be observed that even in case of pessimistic tampering, the additional degree of freedom stemming from the introduced adaptivity can be beneficial, especially for lower quality levels (higher tampering rates).

\section{ViI. Discussion \& Performance Summary}

Existing literature describes numerous self-embedding schemes, characterized by different performance with respect to three fundamental measures: the reconstruction quality, the quality of the protected images, and the boundary tampering rate. Additionally, the schemes might offer uniform and constant reconstruction quality, or adaptive or flexible behavior. Due to the inherent trade-offs between these performance aspects, the preferred approach might be different for various applications.

In Fig. 11 we summarized the performance of state-of-theart self-embedding schemes. The radar plots include the three mentioned fundamental performance measures. The tampering rate is normalized to $60 \%$ of the image area. The quality scores' grid corresponds to $5 \mathrm{~dB}$ changes in PSNR, starting from $20 \mathrm{~dB}$, which we consider the lowest sensible score. The results were obtained on 48 natural images from the BOWS2 data set in a common evaluation scenario [10].

Fig. 11abc compare the performance of traditional uniformquality schemes, including the reference sharing scheme A from [14], the scheme from [13], and the reference scheme $(\lambda=1)$ from [10]. The latter provides only slightly worse quality than [14], and only slightly worse tolerance for tampering than [13]. Hence, it seems to be the most universal of the available traditional schemes. 


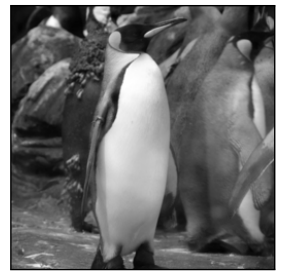

(a) Cover, 4767.png

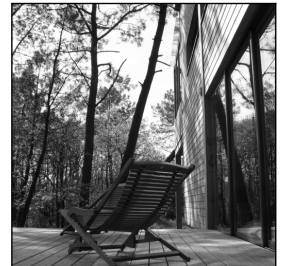

(g) Cover, 9011.png

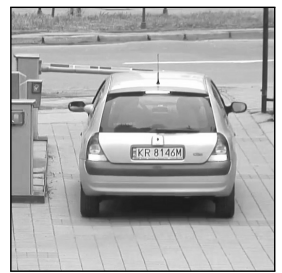

(m) Cover

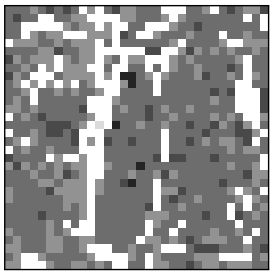

(b) $\tilde{\gamma}=0.30$

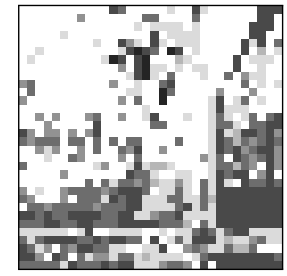

(h) $\tilde{\gamma}=0.30$

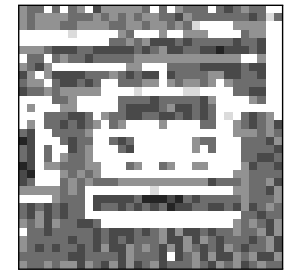

(n) $\tilde{\gamma}=0.30$

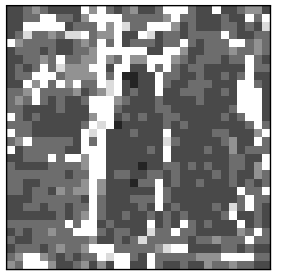

(c) $\tilde{\gamma}=0.35$

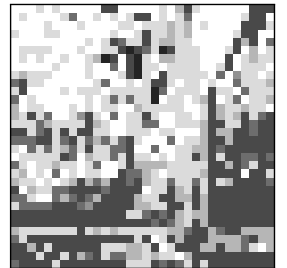

(i) $\tilde{\gamma}=0.35$

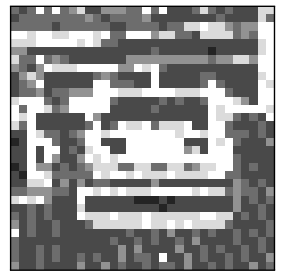

(o) $\tilde{\gamma}=0.35$

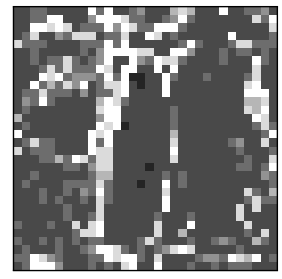

(d) $\tilde{\gamma}=0.40$

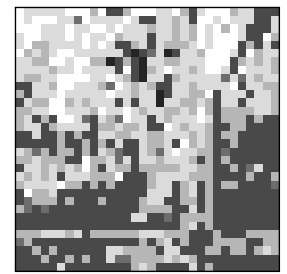

(j) $\tilde{\gamma}=0.40$

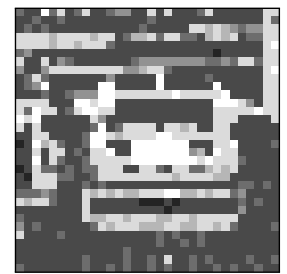

(p) $\tilde{\gamma}=0.40$

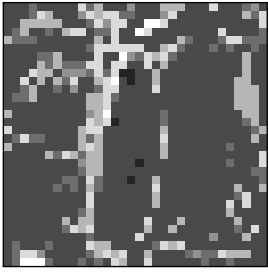

(e) $\tilde{\gamma}=0.45$

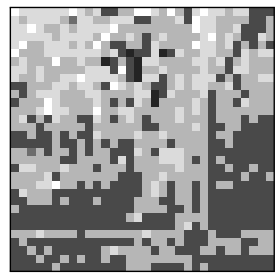

(k) $\tilde{\gamma}=0.45$

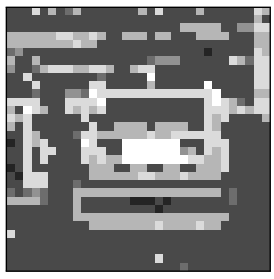

(q) $\tilde{\gamma}=0.45$

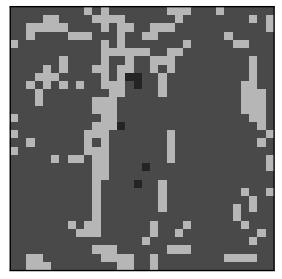

(f) $\tilde{\gamma}=0.50$

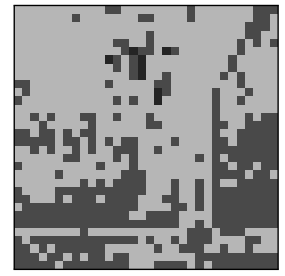

(1) $\tilde{\gamma}=0.50$

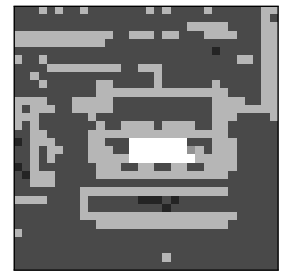

(r) $\tilde{\gamma}=0.50$

Fig. 8: Intermediate descriptors during the design procedure for three example images without (top and middle rows) and with (bottom row) an importance map.

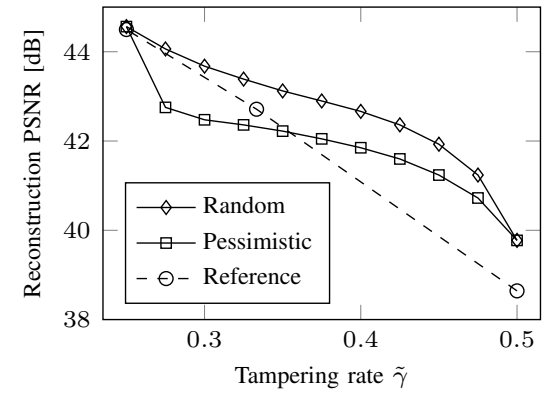

(a) 4767.png

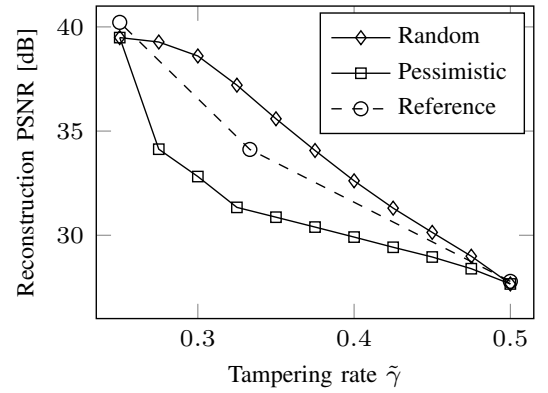

(b) 9011.png

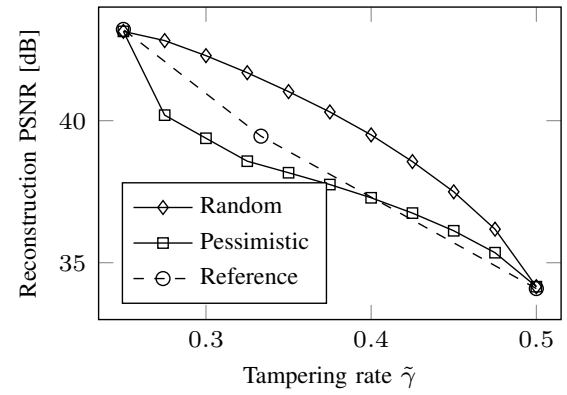

(c) average for 50 images

Fig. 10: Scatter plots of the reconstruction quality vs. the tampering rate for the reference and the adaptive schemes.

Traditional schemes are designed with a specific target performance in mind, e.g., to guarantee reconstruction below a given tampering rate, or to guarantee certain reconstruction fidelity. In the latter case, the tolerance for tampering stems from the selected quality level. In either case, this tolerance is identical for all images. Adaptive schemes give more control over the inherent restoration trade-offs, and allow for choosing the desired performance for each image individually. However, when the actual tampering exceeds the boundary rate, the reconstruction is not possible. One possible approach to address this problem is to employ flexible reconstruction techniques. They deliver high reconstruction quality when the tampering is limited, but their performance tends to deteriorate quickly as the tampering rates grow. While flexible reconstruction mechanisms typically do not have a hard limit on the maximum tampering rate, below a certain threshold the reconstruction result might no longer be discernible.

Due to the fundamentally different approach, it is difficult to directly compare the performance of adaptive and flexible self-embedding. Fig. 11def summarize the overall performance changes for the proposed adaptive scheme, and two flexible schemes [2], [3]. The adaptive scheme is characterized for two extreme boundary tampering rates, $\tilde{\gamma}=0.25$ and $\tilde{\gamma}=0.50$, stemming from (33). The performance for the flexible schemes is shown for actual tampering rates of $\tilde{\gamma}=0.50, \tilde{\gamma}=0.25$, and $\tilde{\gamma}=0.05$.

While the adaptive scheme provides better overall performance, in particular for higher tampering rates, the flexible schemes will improve their reconstruction quality when the actual tampering rate decreases (dashed line in Fig. 11de). In general however, flexible schemes seem to have worse tradeoff capabilities. Hence, despite not being able to fully exploit 


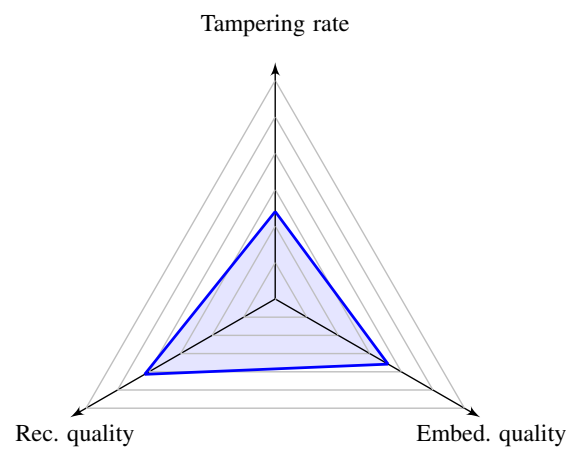

(a) Reference sharing scheme A [14]

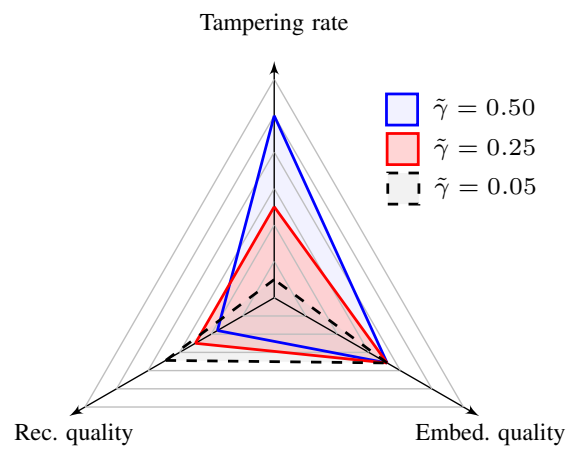

(d) Flexible scheme [2]

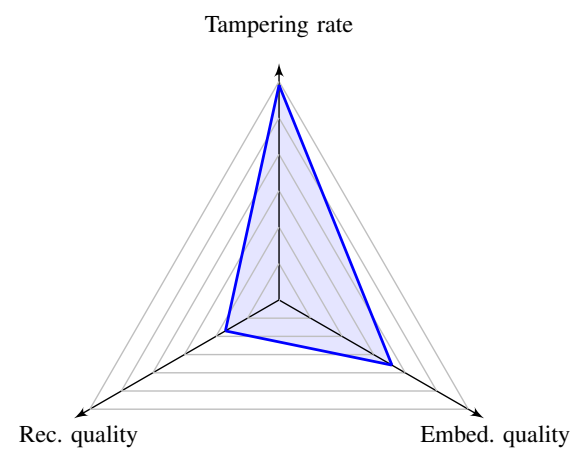

(b) Scheme [13]

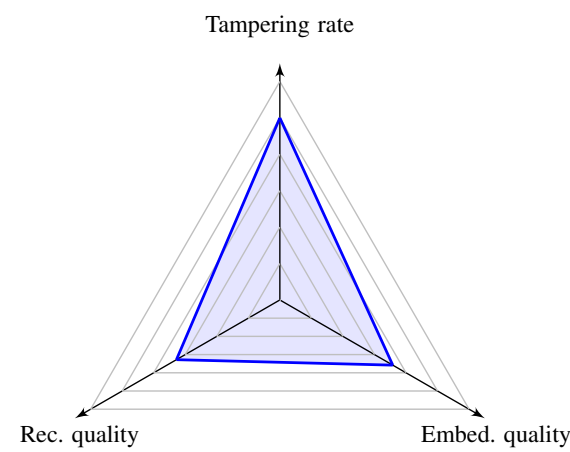

(c) Reference scheme [10], $\lambda=1$

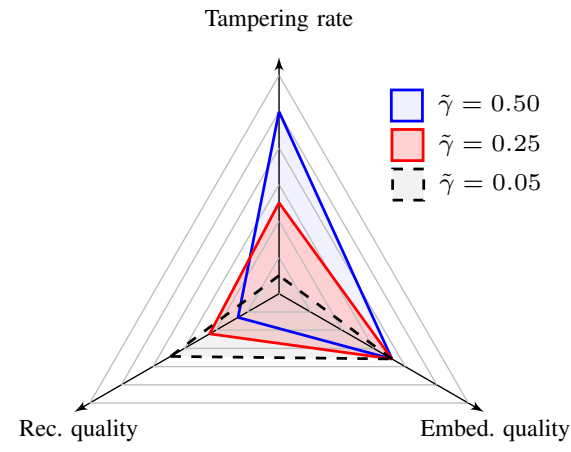

(e) Flexible scheme [3]

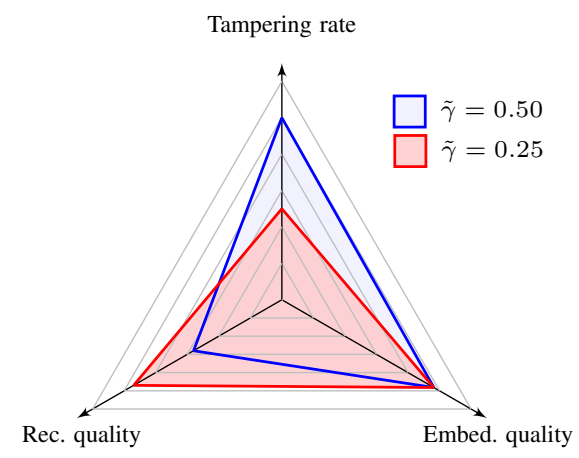

(f) Proposed adaptive scheme

Fig. 11: Summary of various performance aspects of state-of-the-art self-embedding schemes. Tampering rates are normalized to 0.6; quality scores' grid corresponds to changes by $5 \mathrm{~dB}$ in terms of PSNR, starting from $20 \mathrm{~dB}$.

the actual tampering conditions, adaptive schemes still provide a valid alternative.

In many practical applications, a hybrid scheme might actually be preferable. It would be beneficial to guarantee highquality reconstruction for selected image fragments, while allowing for best-effort reconstruction for the remaining, less important content. Development of such a scheme is an interesting research direction.

\section{CONCLUSIONS}

In conclusion, introduction of content adaptivity has an important impact on the achievable tampering rates. Even adoption of reconstruction profiles with lower fidelity does not necessarily lead to improvement in the restoration conditions. Due to more flexibility in controlling the inherent restoration trade-offs, it is possible to obtain better efficiency of the reconstruction process. However, when the tampering affects the most important content, the supported tampering rates are significantly reduced.

Our study shows that the notion of the reconstruction demand can be used to accurately model the behavior of adaptive self-recovery schemes. The derived theoretical formulas can be exploited during computation of the mapping between the reconstruction profiles and image blocks. The presented procedure takes into account the constraints on the local reconstruction quality, and the desired tampering rates. It is possible to provide guarantees for certain aspects of the reconstruction process, which is of high importance in certain applications.

\section{REFERENCES}

[1] Jessica Fridrich and Miroslav Goljan, "Images with self-correcting capabilities," in Proc. of IEEE International Conference on Image Processing, 1999.

[2] Xinpeng Zhang, Shuozhong Wang, Zhenxing Qian, and Guorui Feng, "Self-embedding watermark with flexible restoration quality," Multimedia Tools and Applications, vol. 54, pp. 385-395, 2011.

[3] Xinpeng Zhang, Zhenxing Qian, Yanli Ren, and Guorui Feng, "Watermarking with flexible self-recovery quality based on compressive sensing and compositive reconstruction," IEEE Transactions on Information Forensics and Security, vol. 6, no. 4, pp. 1223-1232, 2011.

[4] Paweł Korus and Andrzej Dziech, "A novel approach to adaptive image authentication," in Proc. of IEEE International Conference on Image Processing, Brussels, 2011.

[5] Zhenxing Qian, Guorui Feng, Xinpeng Zhang, and Shuozhong Wang, "Image self-embedding with high-quality restoration capability," Digital Signal Processing, vol. 21, no. 2, pp. 278-286, Mar. 2011.

[6] Chuan Qin, Chin-Chen Chang, and Pei-Yu Chen, "Self-embedding fragile watermarking with restoration capability based on adaptive bit allocation mechanism," Signal Processing, vol. 92, no. 4, pp. 1137 1150, 2012.

[7] Chuan Qin, Chin-Chen Chang, and Kuo-Nan Chen, "Adaptive selfrecovery for tampered images based on VQ indexing and inpainting," Signal Processing, vol. 93, no. 4, pp. 933 - 946, 2013.

[8] Yaoran Huo, Hongjie He, and Fan Chen, "Alterable-capacity fragile watermarking scheme with restoration capability," Optics Communications, vol. 285, pp. 1759-1766, 2012.

[9] Zhenxing Qian and Guorui Feng, "Inpainting assisted self recovery with decreased embedding data," IEEE Signal Processing Letters, vol. 17, no. 11, pp. 929-932, Nov. 2010.

[10] Paweł Korus and Andrzej Dziech, "Efficient method for content reconstruction with self-embedding," IEEE Transactions on Image Processing, vol. 22, no. 3, March 2013. 
[11] Xunzhan Zhu, Anthony T.S. Ho, and Pina Marziliano, "A new semi fragile image watermarking with robust tampering restoration using irregular sampling," Signal Processing : Image Communication, vol. 22, no. 5, 2007.

[12] Xinpeng Zhang and Shuozhong Wang, "Fragile watermarking with error free restoration capability," IEEE Transactions on Multimedia, vol. 10, no. $8,2008$.

[13] Xinpeng Zhang, Shuozhong Wang, and Guorui Feng, "Fragile watermarking scheme with extensive content restoration capability," in Proc. of International Workshop on Digital Watermarking, 2009.

[14] Xinpeng Zhang, Shuozhong Wang, Zhenxing Qian, and Guorui Feng, "Reference sharing mechanism for watermark self-embedding," IEEE Transactions on Image Processing, vol. 20, no. 2, pp. 485-495, 2011.

[15] David J. MacKay, Information Theory, Inference, and Learning Algorithms, Cambridge University Press, 2003.

[16] David J. MacKay, "Fountain codes," IEE Proceedings Communication, vol. 152 , no. $6,2005$.

[17] "The dataset from the 2nd bows contest," http://bows2.ec-lille.fr/, 2007, Visited on 26 March 2012.

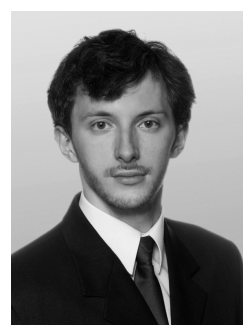

Paweł Korus received his M.Sc. and Ph.D. degrees in telecommunications (both with honors) from the AGH University of Science and Technology in 2008, and in 2013, respectively.

$\mathrm{He}$ is currently an assistant professor at the Department of Telecommunications of the AGH University of Science and Technology, Krakow, Poland. Since 2008 he has actively participated in both national and international research projects, e.g., BONE, INDECT, and INSIGMA. His research interests include multimedia security, digital watermarking, information hiding, and multimedia communication systems.

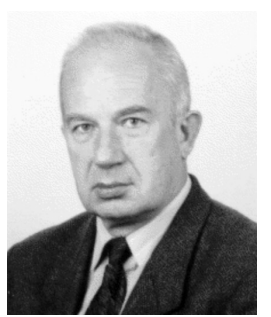

Andrzej Dziech holds the position of a full professor at the Department of Telecommunications of AGH University of Science and Technology in Krakow, Poland.

He received his M.Sc. and Ph.D. degrees from the Institute of Electrical Engineering in Saint Petersburg in 1970 and 1973, respectively, and the D.Sc. from Technical University of Poznan in 1978. He is an author of 6 books and nearly 180 publications. $\mathrm{He}$ was a supervisor of $18 \mathrm{Ph} . \mathrm{D}$. students.

His fields of interest are related to digital communication, image and data processing, data compression, information and coding theory, random signals, computer communications networks and signal processing. He was awarded 4 times for research achievements by the Ministry of Education of Poland. Professor Dziech actively participated in numerous international research projects, e.g., Tempus, Knixmas, Calibrate. Currently, he is coordinating a European Union FP7 integrated project INDECT. 